\title{
INTERCAMBIO ONOMÁSTICO ENTRE JUDÍOS Y CRISTIANOS EN EL REINO DE NAVARRA EN LA ÉPOCA MEDIEVAL: APUNTES HISTÓRICO- LINGÜÍSTICOS
}

ONOMASTIC EXCHANGE BETWEEN JEWS AND CHRISTIANS IN THE KINGDOM OF NAVARRE DURING THE MEDIEVAL PERIOD: HISTORICALLINGUISTIC NOTES 
El presente estudio se propone medir hasta qué punto conocían la lengua vasca los judíos que habitaban el reino de Navarra en época medieval. Los judíos navarros hablaban romance y hebreo, de lo que han dejado abundante testimonio escrito. No ocurre así con la lengua vasca, ya que no hay un documento que constate de forma contundente su uso por parte de los judíos. Sin embargo, se hacían nombrar y apodar a menudo con nombres y sobrenombres vascos. Nuestro propósito es, aproximándonos a las fuentes desde otra perspectiva, demostrar una familiaridad con la lengua vasca que compartían con sus vecinos cristianos. A través de la ciencia onomástica obtendremos información inaccesible por otros medios, y pondremos atención especial en una característica común entre judíos y cristianos. En lugar de señalar los rasgos distintivos que hacen identificable a esta minoría a lo largo de su historia, señalamos un rasgo común con la mayoría cristiana que revela un grado de integración considerable dentro de esta.

Palabras Clave: onomástica, judíos, Navarra, Edad Media, lengua vasca

The essential starting point of this study has to do with the fact, that Jews in Medieval Navarre knew and, in all probability, spoke not only Romance and Hebrew, but also Basque. Their use of Hebrew and Romance is easily demonstrable through documents written by Jews in those languages. Unfortunately, there is no evidence of any document written in Basque by a Jew. My tentative contention is that, approaching the fonts from another perspective, we can see that they indeed were familiar with Romance of course, but also with Basque. Instead of looking for characteristics that made Jews identifiable throughout Navarrese history, - distinguishing them from the Christian majority-, we have paid attention precisely to a feature they shared with Christians: the familiarity with the Basque language. In brief, an element common to both Jews and Christians in medieval Navarre that made of this minority an integrant part of the society where they lived after generations.

Key wORDs: onomastics, jews, Navarre, Middle Ages, Basque language

FECHA DE RECEPCIÓN: 15/08/2015

FECHA DE RECEPCIÓN: 22/09/2015 


\section{INTERCAMBIO ONOMÁSTICO ENTRE JUDÍOS Y CRISTIANOS EN EL REINO DE NAVARRA EN LA ÉPOCA MEDIEVAL: APUNTES HISTÓRICO- LINGÜÍSTICOS*}

\section{ONOMASTIC EXCHANGE BETWEEN JEWS AND CHRISTIANS IN THE KINGDOM OF NAVARRE DURING THE MEDIEVAL PERIOD: HISTORICAL- LINGUISTIC NOTES}

Eunate Mirones Lozano Universidad de Salamanca

La onomástica medieval se estudia hace mucho tiempo con empeño y dedicación desde diversas perspectivas, con objetivos bien distintos, y resultados a menudo notables. Esta es una de las principales razones que nos mueve a llevar a cabo el presente estudio. En él se aplicará la ciencia onomástica al caso concreto de la comunidad judía navarra en la época bajo medieval. Se fijará la atención en una serie de testimonios onomásticos muy particulares, cuyo interés reside en su excepcionalidad y capacidad informativa. Estos ofrecen una información flexible, versátil y aplicable a muy diferentes propósitos, y especialmente adecuada en el caso que nos ocupa.

* Agradezco a los revisores anónimos las sugerencias hechas tras su atenta lectura del texto, que han contribuido notablemente a su mejora. 
La perspectiva desde la que nos aproximamos es histórico-lingüística. El objetivo que se persigue se fija, a grandes rasgos, en la obtención de datos que permitan precisar niveles de integración y convivencia de la minoría judía dentro de una comunidad mayoritariamente cristiana. Para alcanzar este objetivo nos proponemos ampliar la información que ya tenemos sobre onomástica judía medieval en suelo navarro con noticias no suficientemente conocidas al respecto.

En el apartado que las monografías sobre comunidades judías medievales dedican a la onomástica se sigue, por lo general, un mismo protocolo. En primer lugar, se recorren los nombres que aparecen documentados de la comunidad objeto de estudio y se establece su procedencia y, cuando es posible, se señalan las familias de mayor importancia. A partir de ahí, se procura interrelacionar los datos obtenidos con el resto de informaciones derivadas del estudio de otros aspectos de dicha comunidad. En ocasiones, las menos, es posible recoger la trayectoria del apellido de una familia a lo largo de generaciones. Un ejemplo de ello es el estudio que realiza Beatrice Leroy de la reputada familia Menir de origen tudelano. En él se estudia con detalle los avatares de esta familia desde el siglo XII en adelante. La autora, siguiendo a los Menir en su devenir, nos da a conocer sus circunstancias al tiempo que nos informa sobre la comunidad judía en cuyo seno vivieron a lo largo de muchas generaciones: primero en Navarra y más tarde emigrados a distintos lugares tras la expulsión de la Península (Leroy, 2001).

Los estudios como el mencionado son raros, ya que es difícil que los documentos preservados permitan seguir a una familia a lo largo de los siglos como en el caso que 
estudia Leroy. Por lo general, el apartado dedicado a la onomástica en las monografías sobre la historia de los judíos navarros suele ser un alto en el camino más bien formal y con peso relativo dentro del conjunto del estudio. ${ }^{1}$ En esta ocasión queremos dar un paso más y detenernos en un aspecto muy concreto, que nos ha interesado de forma especial y del que no se ocupa ninguna de las monografías al uso: los sobrenombres ${ }^{2}$ de judíos que aparecen escritos en lengua vasca. Creemos que el uso de esta lengua para formar sobrenombres da, si bien de forma indirecta, una información valiosa acerca de la convivencia e integración de los judíos dentro de la comunidad cristiana del reino de Navarra.

\section{Contexto geográfico-temporal}

El espacio geográfico que se quiere abarcar es aquel que queda dentro de las fronteras de lo que era el reino de Navarra en época bajo medieval. Más en concreto el territorio perteneciente a dicho reino en el periodo que va desde el año 1349 hasta 1479. En 1349 comienza el reinado de Carlos II, al que sucede en 1387 Carlos III el Noble. Este último reina en Navarra hasta 1425, año en que es sucedido por su hija Blanca, que muere en 1441, quedando el reino en manos de

\footnotetext{
1 Tres monografías a consultar en este sentido son: Leroy, 1991; Gampel, 1989; Mirones Lozano, 1999.

${ }^{2}$ Entendemos por 'sobrenombre', tal y como lo define la RAE: bien el 'nombre que se añade a veces al apellido para distinguir a dos personas que tienen el mismo'; o bien el 'nombre calificativo con que se distingue especialmente a una persona'.
} 
su marido Juan II hasta su muerte en 1479. A este periodo pertenecen la mayor parte de los documentos que utilizamos; si bien haremos uso de algunos testimonios anteriores en el tiempo para completar el análisis.

Somos conscientes de que se trata de un espacio físico y temporal demasiado amplio. La razón de decidirse por estos límites tan extensos es que los testimonios que nos interesan, aunque no excepcionales, son poco comunes. Lo más frecuente era que los judíos navarros se identificaran con nombre y apellidos típicamente judíos. De no ser así, se hacían llamar por un nombre inequívocamente judío, al que seguía un apellido, o apodo ${ }^{3}$ según los casos, en romance navarro u occitano. Sin embargo, los testimonios onomásticos en lengua vasca preservados no son muy abundantes. Por consiguiente, hemos decidido abarcar un espacio temporal extenso que nos permita obtener testimonios en número y calidad suficiente para poder llevar a cabo un examen de interés.

El problema de delimitar el trabajo de este modo es que imposibilita, en esta fase, un estudio del todo completo. Para completarlo habría que analizar los antedichos testimonios en lengua vasca comparándolos con sus análogos en romance para el mismo periodo y espacio geográfico. Luego, una vez recopilados unos y otros, contrastar y analizarlos en conjunto. Llevar a cabo este trabajo se convertiría en una in-

\footnotetext{
${ }^{3}$ La RAE define apodo como el "nombre que suele darse a una persona, tomado de sus defectos corporales o de alguna otra circunstancia”. En los ejemplos que nos ocupan apodo y sobrenombre son prácticamente sinónimos; de ahí que intercambiemos ambos vocablos a lo largo del estudio.
} 
vestigación demasiado extensa para un artículo como el que se presenta, pero que no se descarta realizar en un futuro.

Descartamos, de momento, la alternativa lógica de reducir el contexto geográfico-temporal porque, como hemos dicho, si así lo hiciéramos, no podríamos contar con suficientes testimonios de sobrenombres en lengua vasca para enunciar hipótesis alguna. Así pues, en este primer acercamiento a la cuestión, consideramos este amplio contexto geográfico-temporal para poder reunir una serie digna de documentos que posibilite un primer análisis. En un estudio ulterior se quiere trabajar la documentación cristiana para la misma época y región.

\section{Fuentes documentales}

Las fuentes documentales que se utilizan son las conservadas en el Archivo General de Navarra. Se trata de documentos de carácter eminentemente fiscal que refieren básicamente las cuentas del reino y a aquellos implicados en las mismas. Se han consultado sobre todo los libros del procurador fiscal, y los comptos reales conservados para este periodo. Estos manuscritos tienen las limitaciones propias de este tipo de documentación; parca en noticias sobre aquellos que aparecen mencionados, más allá de sus nombres y apellidos, y en ocasiones su profesión. Sin olvidar, por otro lado, las reservas propias a las que obliga el material que se maneja, que no es más que una muestra muy fragmentaria de la realidad. Ahora bien, precisamente por ser los nombres y apellidos lo único que aparece siempre en los documentos, por breves 
y sucintos que estos sean, es por lo que se hace posible un estudio onomástico como el que se presenta.

El periodo histórico que contempla el estudio es por lo demás muy inestable. Inestabilidad que queda reflejada en los papeles del reino y que hace que una parte importante de la documentación resulte difícil de revisar. A nuestro favor juega el impecable estado de la colección documental protagonista de nuestras consultas, ya que el Archivo General de Navarra es uno de los mejor conservados y organizados para este periodo de la historia a nivel de toda la península.

\section{Objeto de estudio y razón de ser del artículo}

El objeto de estudio es la comunidad judía que habitaba el suelo navarro en el periodo y espacio establecidos al principio de este escrito. La razón de ser del artículo, como se apuntaba igualmente, es la aportación de nuevos datos onomásticos que, sumados a la información onomástica y de otra naturaleza ya existentes, ayuden a precisar cuál era el nivel de integración de dicha comunidad en el seno de la mayoría cristiana de Navarra en la baja Edad Media. Es decir, queremos añadir noticias que ayuden a perfilar de forma más clara la vida de esta comunidad en relación con sus vecinos cristianos. Con este fin consideraremos como un posible indicador de integración las distintas lenguas que se hablaban en el reino navarro entonces y el uso que hacían de ellas los judíos navarros.

Los niveles de integración y convivencia de esta minoría en la sociedad cristiana de la época se miden en función de 
varios factores: las profesiones a las que se dedican los judíos; su reparto geográfico en el reino; el número de hogares judíos establecidos en una zona determinada; y su participación en las cuentas del reino. Describir a la comunidad judía tan solo con esta información es tarea difícil y, por lo general, quedan muchos espacios en blanco por dilucidar. Es con ánimo de completar una parte, aunque modesta, de estos espacios en blanco, por lo que se ha decidido ir un poco más allá y analizar la realidad plurilingüe del reino navarro a través de los nombres. La utilización por parte de la minoría judía de las lenguas vernáculas habladas entonces en el reino navarro podría ser un indicador de convivencia; y de forma especial la lengua vasca por las razones que daremos más adelante.

\section{Realidad plurilingüe del reino navarro}

En este periodo de la historia de Navarra se hablaban en el reino principalmente dos variedades románicas: el romance navarro y el occitano. El romance navarro se hablaba en las principales poblaciones del reino. La otra variedad románica, procedente del otro lado del Pirineo, era el occitano común, cercano al languedociano y circunscrito a los burgos de San Cernín y San Nicolás de Pamplona, de San Martín y San Juan de Estella, Puente la Reina y Sangüesa. Sus primeras muestras escritas son coetáneas de las del romance navarro, a partir del 1200, y lo escribirán los funcionarios de la chancillería real hasta el siglo XIV. Ahora bien, la lengua administrativa de la corte, que emplearán los notarios y 
gentes libres de las villas desde principios del siglo XIII, será principalmente el romance navarro.

Aparte de estas dos variedades de romance se hablaba en el reino de Navarra también la lengua vasca. ${ }^{4}$ En ella se expresaban sobre todo los pequeños labradores pecheros, collazos y pastores de la mitad norte del reino que constituían casi dos tercios de la población total del reino. Sin embargo, esta lengua no se escribía prácticamente, ya que la población que la hablaba era mayoritariamente ágrafa, por lo que los testimonios escritos en vasco son escasísimos para este periodo. ${ }^{5}$ Se ha dicho durante mucho tiempo que subsistió sin escribirse por habitar sus hablantes tierras de difícil acceso con pocos recursos económicos; aunque esta opinión ha sido largamente rebatida hace tiempo. ${ }^{6}$

Por fin, las dos minorías del reino, la judía y la musulmana, hacían uso, junto con el romance, de sus lenguas: el hebreo y el árabe. De ambas lenguas hay testimonios escritos, ${ }^{7}$ y las dos quedan igualmente reflejadas en la ono-

\footnotetext{
${ }^{4}$ La lengua vasca suscita mucho interés y ha dado lugar a numerosos trabajos que se ocupan de su descripción e historia. Recomendaría por su calidad y exhaustividad la obra de Trask (1997). En este trabajo sintetiza con rigor muchos de los estudios que le han precedido de autores fundamentales como J. Lakarra o J. I. Hualde, que se han ocupado de aspectos más específicos del euskera. No son los únicos; pero la lista de estudiosos de la lengua vasca cuya lectura es aconsejable es demasiado larga para referirla aquí. De muy obligada mención y consulta es la obra filológica de Koldo Michelena, algunos de cuyos trabajos se mencionarán más adelante.

${ }^{5}$ Los testimonios que se han conservado no van más allá de unas frases de correspondencia privada entre dos funcionarios reales en un recibo de las cuentas reales; y una brevísima anotación en otro documento del mismo tipo (Irigaray, 1970: 135-136; Idoate, 1969: 287-290; Alegría, 2002: 139-148).

${ }^{6}$ Este asunto lo trata en profundidad Jimeno Jurío (1997a: 33).

${ }^{7}$ Todos los documentos preservados escritos en lengua hebrea y aljamía hebraicoespañola se encuentran recogidos en el trabajo de Lacave (1998).
} 
mástica. El árabe, de hecho, se identifica, no solo en los nombres de la población musulmana; también en ocasiones en los nombres de los judíos. Concretamente en los nombres de aquellos judíos emigrados al norte desde los territorios musulmanes y sus descendientes. Estos llevaban con frecuencia la forma arabizada de algunos nombres como Abraham, "Ibrayim” en su versión árabe. E incluso, aunque el primer nombre de los judíos navarros en el caso de los hombres es casi siempre judío, podía ser excepcionalmente árabe, como por ejemplo "Galaf”, una variante del árabe "Halif”, 'califa, sucesor' (Becker, 2009: 147).

\section{Los nombres de los judíos navarros}

Esta realidad plurilingüe presente en el reino navarro en la Edad Media, se expresa en la onomástica tanto cristiana como judía. La onomástica judía, en la que están representadas todas las lenguas de las que hemos hablado, es la que nos interesa. Por un lado, como acabamos de hacer notar unas líneas más arriba, se puede rastrear en ella el árabe. Se dan también casos de sobrenombres que denotan origen ultra pirenaico. Su presencia en las fuentes se revela especialmente a raíz de la expulsión de judíos del territorio francés en 1306. Como consecuencia de la expulsión, las aljamas

La documentación en árabe es más escasa. Sin embargo, a pesar de haber pocos testimonios, García Arenal apuntó hace tiempo que, hasta el momento de su conversión en 1516, se constata cierta utilización de la lengua árabe por los moros, al menos los de Tudela, donde se concentraba el mayor número de esta población. De esto la autora da pruebas documentales inequívocas (1982: 37). 
del reino de Navarra acogerán emigrantes judíos del otro lado del Pirineo, y esto quedará reflejado en los documentos del reino, en concreto en los libros de pagos que años después los judíos venidos de tierra de Ultrapuertos harán a la Corona. ${ }^{8}$ Por fin, los sobrenombres expresados en romance navarro son, sin lugar a dudas, los más comunes; los más comunes después de los que son característicamente judíos obviamente. Y por último tenemos testimonios de sobrenombres en lengua vasca, que son los que interesan al estudio y de los que hablamos en detalle a continuación.

\subsection{Los sobrenombres vascos}

La lengua vasca es lengua de indudable interés por muchas razones, lo que se refleja en debates persistentes sobre cada uno de sus aspectos. Debates en los que no entraremos, puesto que lo que nos interesa aquí de esta lengua no es objeto de discusión alguno. Este trabajo fija su atención en una serie de testimonios documentales de judíos navarros con sobrenombres en lengua vasca, ${ }^{9}$ cuyo valor no radica en lo que de ellos pueda aprenderse sobre la legua vasca. Lo que interesa al estudio es la posibilidad de que el uso

\footnotetext{
8 Juan Carrasco estudia un documento con el nombre de 'pecha de los judíos de Ultrapuertos' en la que aparecen listados una serie de judíos cuyos apellidos denotan su origen ultra pirenaico: "Calot", "Bonanic", "Bienvengut", "Tunenex", etc. (1993: 342). Es interesante consultar los artículos de Orpustan sobre antroponimia medieval en el País Vasco para conocer más sobre nombres y apellidos atestiguados en lengua occitana en época medieval (2000 y 1997).

${ }^{9}$ Nuestro trabajo se ocupa de una parte muy pequeña de la onomástica judía medieval del reino navarro. Hay estudios que han tratado la onomástica navarra en su conjunto cuya consulta es fundamental: Irigaray, 1960; Cierbide Martinena, 1996: 87-96; 2003; Lacarra, 1930.
} 
del euskera para nombrarse pueda considerarse como un indicador significativo de integración en una sociedad a la que pertenecen desde tiempos remotos. De ser así, podría considerarse, además, como un rasgo de interés susceptible de enriquecer cualquier estudio de alteridad que trate las diferencias y afinidades de esta minoría dentro del conjunto de la mayoría cristiana (Valdeon, 2006; Niremberg, 2001; Riera i Sans, 2009).

Creemos, de entrada, que el hecho de que los judíos utilicen la lengua vasca para nombrarse señala un lazo muy estrecho con el territorio navarro. Otorgarse un nombre no es, ni ha sido nunca, un gesto arbitrario en cultura alguna conocida. Muy al contrario, es un acto definitorio que se cuida mucho y raramente se deja al azar. Por otro lado, la lengua vasca tiene un componente de arraigo a la tierra indiscutible, que poco o nada tiene que ver con su escasa tradición escrita. En el caso que nos ocupa el uso de esta lengua para nombrase, y no otra, es una forma de expresión manifiesta de integración y convivencia de una comunidad minoritaria en un entorno mayoritariamente cristiano, en este caso plurilingüe. Muestra sin lugar a dudas un grado más de vinculación con la tierra que habitan y sus naturales, que se puede sumar a los indicadores que tradicionalmente miden la convivencia entre comunidades.

El uso de sobrenombres en lengua vasca no da necesariamente testimonio de su conocimiento efectivo. Es obvio que uno puede llevar un sobrenombre sin conocer su significado; luego difícilmente tal uso será síntoma inequívoco del conocimiento de una lengua. Así pues, el nombrarse de esta manera no demuestra necesariamente que habla- 
ran vasco, ni siquiera que entendieran los apellidos o sobrenombres que les titulaban. No obstante, nos inclinamos a pensar que probablemente los entendían, porque en más de un ocasión - sobre todo en el caso de los apodos-, todo parece indicar que el judío que aparece en el documento es con toda probabilidad uno de sus primeros portadores. Sería extraño que uno no entienda el sobrenombre con el que le identifican siendo el primero que lo acuña.

Hablamos aquí del caso concreto en que el apodo sigue al nombre completo del judío, aún no transformado en apellido, como puede ser el caso de "Judas Alborge, dicto Ederra". Ederra ('hermoso') acabará por ser un apellido, sin embargo aquí lo encontramos todavía en estadio de sobrenombre o apodo. Siendo así, parece difícil creer que el portador, con toda probabilidad el primero, no entendiera el sobrenombre con el que le identificaban. Cuando estos apodos se transforman en apellidos, al pasar de padres a hijos a lo largo de generaciones, pueden empezar a funcionar tan solo como identificadores que no refieren ya ninguna característica particular de su portador y que, en ocasiones, carecen de significado para el que lo lleva. Sin embargo, para que esto ocurra, para que el apodo pierda por completo su sentido original y ya nadie comprenda lo que significa, han de pasar muchos años y no parece que sea lo que ocurre en los casos que nos ocupan.

Con todo, no es posible demostrar de forma incontestable que los judíos en algún momento de su historia en suelo navarro utilizaran efectivamente la lengua vasca para comunicarse. Mucho menos se puede apuntar la posibilidad de una judeo-lengua como las que estudia Magdalena Nom de Deu para otros lugares de la península, en concreto el 
caso catalán, riquísimo en documentación que atestigua su uso (1988-1989; véase también Riera I Sans, 2008). En cualquier caso, la lógica nos empuja a pensar que habría judíos navarros - no olvidemos que eran judíos sí, pero también navarros-, con un conocimiento mayor o menor de la lengua vasca. Es también probable que, dependiendo de la zona geográfica del reino, el oficio, y la misma necesidad según los casos, hubiera judíos que se comunicaran ocasionalmente en esta lengua con vecinos cristianos vascoparlantes. No es un desatino, en fin, pensar que en algún momento los judíos, por diversos motivos o intereses, tuvieran la necesidad de comunicarse en vasco.

Es cierto que no era la lengua oficial ni mayoritaria del reino, pero tenía presencia y arraigo. Es muy posible que determinados habitantes del reino la tuvieran como su primer medio de expresión, resultándoles difícil comunicarse en romance con fluidez. Este hecho queda en cierta medida confirmado por un testimonio documental recogido en años posteriores a los que abarca este estudio. En él, algunos vecinos de un pueblo de la Merindad de Estella, Cirauqui, situado en la zona noroeste del reino, parecen tener problemas para comunicarse en una lengua distinta de la vasca. ${ }^{10}$

El hecho de que la población judía se asentara en su mayor parte en núcleos de población importantes y raramente en pequeñas aldeas de zonas montañosas, ${ }^{11}$ donde la lengua

${ }^{10}$ Muestra de ello es un estudio de Jimeno Junó (1997) sobre el pueblo de Cirauqui.

${ }^{11}$ Para conocer la distribución de la población judía en esta época conviene consultar los siguientes estudios: Leroy, 1978; Gampel, 1989; Carrasco, 1993; Mirones Lozano, 1999. 
vasca era de uso más corriente, pudiera disuadirnos de la posibilidad que apuntamos. Sin embargo, no hay que olvidar que esta población de habla vasca, que se concentra en la parte más montañosa e inaccesible del reino, va a descender paulatinamente a la planicie a lo largo de la Edad Media en busca de mejores condiciones económicas; y sus pobladores llevarán consigo su lengua. ${ }^{12}$

Así pues, la posibilidad de que los judíos navarros estuviesen familiarizados con la lengua vasca y que la utilizaran queda en suposición, altamente probable, pero suposición; puesto que no se conserva documentación que lo acredite de forma irrefutable. Ya hemos dicho que los testimonios escritos en lengua vasca para esta época son muy raros. Son muy raros de mano cristiana; pretender encontrarlos de mano judía sería una quimera. No se podría nunca demostrar el uso que los judíos hacían o no de esa lengua por medio de documentos escritos por ellos mismos en vasco. ${ }^{13}$ Sin embargo, el hecho de que no existan documentos de este tipo, o no se hayan conservado en caso de existir, no es tampoco razón suficiente para afirmar taxativamente que no tuviera lugar el intercambio lingüístico del que hablamos.

En este sentido consideramos que los testimonios que aportamos a continuación, dependiendo de la interpretación que se haga de ellos, son evidencias valiosas a la hora

\footnotetext{
${ }^{12}$ Son muchos los trabajos realizados hasta la fecha para entender la evolución de la lengua vasca y su desplazamiento dentro de la geografía navarra y tierras colindantes. Como introducción a ello son de interés, entre otros, los siguientes: Michelena, 1977; Lafon, 1973; Haritschelhar, 1983; Trask, 1997.

${ }^{13}$ Es interesante leer la breve reflexión que hace Gold a este respecto, si bien creemos que malinterpreta en parte el artículo de Magdalena Nom de Deu que utiliza como punto de partida para su trabajo (2003: 537-540).
} 
de argumentar a favor de una más que posible familiaridad con la lengua vasca por parte de la minoría judía del reino navarro. Es difícil creer que se trate únicamente de la adopción automática por parte de los judíos de sobrenombres vascos usados por los cristianos. Nos inclinamos más bien a pensar que optaban por el sobrenombre eusquérico con plena conciencia de hacerlo, sencillamente porque les resultaba natural.

\section{Testimonios documentales}

En los testimonios documentales revisados ${ }^{14}$ encontramos con especial profusión el sobrenombre "Ederra" ('hermoso, bello'). Salvo en pocos casos, como el de "Judas Alborge,

\footnotetext{
${ }^{14}$ La mayor parte de los documentos que se citarán se encuentran en la serie Navarra Judaica que la Universidad Pública de Navarra y el Gobierno de Navarra publican conjuntamente hace años. J. Carrasco, F. Miranda, E. Ramírez Vaquero, Navarra Judaica. Documentos para la historia de los judíos del reino de Navarra: Documentos 1093-1333, vol. 1, Pamplona, 1995 (en lo sucesivo citado Navarra Judaica, 1); Ibíd., Documentos 1334-1350, vol. 2, Pamplona 1997 (en lo sucesivo citado Navarra Judaica 2); Ibíd., Documentos 1351-1370, vol. 3*, Pamplona 1996 (en lo sucesivo citado Navarra Judaica $3^{\star}$ ); Ibíd., Documentos 1371-1386, vol. $3^{\star *}$, Pamplona 1998 (en lo sucesivo citado Navarra Judaica $3^{\star * *}$ ); Ibíd., Regestas e índices de nombres propios, vol. $3^{* * *}$, Pamplona 1998 (en lo sucesivo citado Navarra Judaica $3^{* * *}$ ); Ibíd., Registros del Sello 1339-1387, vol. 4, Pamplona 1996 (en lo sucesivo citado Navarra Judaica 4) ; Ibíd., Registros del Sello 1364-1400, vol. 5, Pamplona 2002 (en lo sucesivo citado Navarra Judaica 5); Ibíd., Registros del Sello 1400-1406, vol. 6*, Pamplona 2003 (en lo sucesivo citado Navarra Judaica 6*); Ibíd., Registros del Sello 1406-1413, vol. 6**, Pamplona 2003 (en lo sucesivo citado Navarra Judaica $6^{* *}$ ); M. Zubillaga publica, siempre en la misma serie, los documentos sobre judíos encontrados en los Protocolos notariales de Cascante (Zubillaga, Navarra Judaica. Los judíos del reino de Navarra. Protocolos notariales de Cascante 1436-1496, vol. 12, Pamplona 2003 que en lo sucesivo será citado Navarra Judaica 12).
} 
dicto Ederra", ${ }^{15}$ mencionado en párrafos anteriores, "Ederra" funciona ya, la mayor parte de las veces, como apellido más que como sobrenombre o apodo. Encontramos también testimonio de su forma hebrea "Jaffe", ${ }^{16}$ e igualmente de su equivalente romance "Hermoso". ${ }^{17}$ Los testimonios se refieren a personas no coincidentes en el tiempo y tampoco en el lugar de origen. Lo ideal sería que las tres formas se refirieran a una misma persona y así poder hablar de un intercambio real y natural de lenguas - romance, hebreo y vasco-; empero, la distancia temporal y física entre los testimonios no nos permite hacer tales afirmaciones.

En este sentido no se cuenta con testimonios onomásticos concluyentes como los que en ocasiones encontramos para la comunidad cristiana de Navarra. Nos referimos con esto a casos en que el documento muestra un uso casi arbitrario de la forma romance y vasca; dando a entender que una y otra forma son perfectamente comprensibles e intercambiables para los portadores. Un ejemplo muy claro y que ilustra muy bien esto que decimos se encuentra en un recibo firmado por un matrimonio en el que el marido escribe "Pedro de la Puent", mientras que su mujer prefiere la forma

\footnotetext{
${ }^{15}$ Un ejemplo de "Ederra" se encuentra en una cuenta de 1334 de los censos que se pagan por "las plazas de la iuderia" de Pamplona, se menciona a "Salomon Ederra" y a su mujer "Cima" (NJ, 2, 269-241). No mencionamos todos los testimonios de este apellido porque son abundantísimos. En forma de apodo, aún en proceso de convertirse en apellido, aparece como "Judas Alborge, dicto Ederra”, judío de Pamplona (NJ, $3^{\star *}$, 680-679).

16 "Jaffe" aparece mencionado en 1353, en un documento en el que un tal "Abram Jaffe", judío de Estella hace de testigo en un préstamo (NJ, 4, 2-107).

${ }^{17}$ En 1375 Carlos II autoriza al alcalde de la Cort a comprar una cámara y su granero situados en la rúa mayor de la judería de Monreal; dice el documento que estos son propiedad de "Gento Fermoso" (NJ, $\left.3^{\star *}, 685\right)$.
} 
eusquérica del mismo apellido, "Çubico" — ('del puente' en euskera) - , y firma, en consecuencia, como "María Çubico" (Lacarra, 1930: 251). Se trata del mismo apellido escrito en las dos lenguas de uso corriente para los firmantes en el momento de ponerse por escrito sus rúbricas.

No hemos encontrado nada parecido entre los judíos; lo que no quiere decir necesariamente que no tuviera lugar. Normalmente entre los testimonios que presentamos, en el caso de encontrar un apellido en su forma romance y eusquérica, no se refieren a la misma persona. De hecho, generalmente se alejan demasiado en el tiempo y el espacio, como es el caso de "Hermosso, "Ederra y "Jaffe". Con todo, consideramos fundamental señalar cuando tienen lugar las dos realizaciones y en consecuencia las señalamos.

Un ejemplo de estas dos realizaciones lo tenemos en el apellido "Corto", "Motça" en su correspondiente vasco. En su realización romance es menos abundante, tenemos un solo testimonio en 1418, en el que se menciona a "Juce Corto" recogiendo impuestos en Tafalla (Mirones, 1999: 105). El resto de testimonios son en euskera: en un registro de cuentas del baile ${ }^{18}$ de Estella figuran unos pagos hechos a "Juçe Mocha” por "obras feytas" (Navarra Judaica, 1, 138-

\footnotetext{
18 Término de contenido muy genérico en los primeros siglos medievales y cuyo significado se fue precisando con el paso del tiempo. A partir de los siglos $\mathrm{X}$, XI se denominan bailes a los agentes o intendentes del rey o de sus delegados, e incluso de los magnates nobiliarios y eclesiásticos, en los diferentes lugares de su jurisdicción o señorío. En los núcleos de población franca, como Pamplona, Tudela o Estella, la denominación de baile se reservó al responsable de la percepción de los derechos fiscales, aplicándose otros títulos (preboste, almirante, justicia) al delegado local en otros ámbitos jurisdiccionales... (Gran Enciclopedia de Navarra, Pamplona 1990, s.v. baile).
} 
181); en 1260 aparece "Mosse Mocha" pagando una multa al preboste de Olite (Navarra Judaica, 1, 85-91). Casi un siglo después, en 1351, se menciona a otro "Mosse Mocha" que "fue iudgado por la Cort et fue enforcado et sus bienes confisquados al rey" (Navarra Judaica, 3*, 370-171; 376-177). En 1333 se menciona en las cuentas de la lezda ${ }^{19}$ de la carnicería de los judíos de Pamplona a "Galef Moch" de Monreal porque debe dos sueldos "por 28 corderos de lech" (Navarra Judaica, 1, 263-319). Se habla en Tudela de un "mestre Moch", judío físico, que solía tener a censo las casas de los judíos del castillo (Mirones, 1999: 105).

Otro ejemplo de este tipo de apellido con dos realizaciones es "Cordero", "Bildocha" en su versión eusquérica. En 1351 en Estella, en un registro de los bienes de los judíos muertos aparece una mención a "Abraham", fijo de "Judas Leui Cordero" (Navarra Judaica, 3*, 370-167). Su equivalente en euskera se menciona entre los judíos que realizan los pagos al baile de Tudela entre los años 1259 a 1266; se trata del padre de un tal "Açach" al que se identifica como "fil de Bildocha" (Navarra Judaica, 1, 85-57). También encontramos el sobrenombre "Bueno" en romance y su correspondiente en euskera "Ona”. Un tal Bueno, judío de Tudela, hace de testigo de un préstamo en 1358 (Navarra Judaica, 4, 7-49). En 1383 se menciona en Tudela a Algarz de Solbeillita

\footnotetext{
${ }^{19}$ Impuesto que se pagaba al fisco por las operaciones comerciales. Aparece también con la grafía "lezta". A partir del siglo XIII se reservó el nombre de lezda para el impuesto que pesaba sobre las compraventas efectuadas en los mercados, mientras que se utilizó el de peaje para el que gravaba la importación y exportación por las fronteras del reino, y que hasta entonces se denominaba también lezda, con frecuencia... (Gran Enciclopedia de Navarra, s.v. lezda).
} 
'muger de rabi Hona que vende una viña a Hamet Catalan (Navarra Judaica, $\left.3^{* *}, 753-125\right)$.

Hay asimismo otros testimonios de apodos en euskera, aún no transformados en apellido, de los que no hemos encontrado equivalente ni en romance ni en hebreo: "Chacur" ('perro') y "Belcha” ('negro'). A "Simuel Alfaqui dito Chacur", se le menciona en 1444 tributando el "ganado vivo" en Pamplona (AGN, Comptos, Reg. 460, fol. CLXXXVv), y años antes, en 1433, se habla de él porque un bastero cristiano de Pamplona le hiere gravemente (AGN, Comptos, caj. 135, no 30, IV). En 1437 se menciona a otro judío apellidado "Alfaqui", "Salamon" en este caso, citado a instancias judiciales a causa de una deuda que tiene con un mercader cristiano de Pamplona y al que también se apoda "Chacur" (AGN, Comptos, caj. 145, $\mathrm{n}^{\circ}$ 15, III). El otro caso es el de "Juçe Amatu", llamado "Belcha", que en 1429 se encuentra entre los contribuyentes judíos de Monreal participando de la ayuda especial otorgada ese año al rey a causa de la guerra castellana (AGN, Comptos, Papeles Sueltos, Leg. 167, carpeta 33, fol. VIIv).

Otro apellido, inconfundiblemente vasco, del que no hemos encontrado equivalentes ni en romance ni en hebreo es "Çuri” ('blanco'). ${ }^{20}$ Aparece en más de una ocasión: en 1381, en Tudela, "Jento Çuri" vende a "Johan de Lor" una "vynna en Boar por 6 libras" (Navarra Judaica, $3^{\star *}, 753$ 219); "Samuel Çuri”, que casa con "Jamila”, hija de "Azac ben

\footnotetext{
${ }^{20}$ Probablemente los Suri de los que habla David Romano en un artículo sobre los judíos de Huesca, tengan algo que ver con los "Çuri" de los que hablamos aquí (1980).
} 
Bibach" (Lacave, 1998: 49) y que aparece mencionado en una ketubah ('contrato matrimonial') de 1309; también hay testimonio de un "Çahadia Çuri", vecino de Tudela, como testigo de un préstamo en 1352 (Navarra Judaica, 4, 3-149). Encontramos por fin en un mismo documento a "Abraham", "Simuel Yento" y "Juce Zuri" todos ellos vecinos de Corella en el registro del sello de Tudela que tenía "Johan Caritat" (Idoarte, 1964: 994).

"Mehe" ('delgado'), ${ }^{21}$ como los anteriores no tienen correspondientes romances o hebreos. Hay un "Abraham Mehe", probablemente residente en Estella y cuyo libro de cuentas publica Lacave (1998: 21), y antes que él, Assis y Magdalena Nom de Deu (1992). En 1323 se menciona otro "Mehe", en este caso "Juçe Mehe", cuya casa junto con la de otros vecinos se usa de referencia para determinar los lindes de una propiedad en el término de Carcalaseta, también en Estella (Lacave, 1998: 6-3). En un documento de 1436 se menciona a dos judíos de Cascante, "Rael" y "Azac Mee", padre e hijo, a los que "Abraham Abendanon" debe 12 florines de oro (Navarra Judaica, 12, 2). En 1381 "Gento Mehe" vende "una vinna en Val de Baron" a "Garcia Gil de Sacristan" por 60 sueldos y "una pieça en Perezuelas" a "Pero Garcia" por 7 libras, 10 sueldos (Navarra Judaica, $3^{\star *}, 743-346 / 7$ ).

Una sola vez aparece un judío denominado "Ossaua" ('tío'), del que existe correspondiente en romance pero no entre los judíos. Con todo, nos ha parecido importante mencionarlo. En una recepta de dineros por homicidios y calo-

\footnotetext{
${ }^{21}$ Michelena, 1987: s.v. mehe: 'delgado, fino, flaco'. Sobre este sobrenombre en concreto interesa la consulta del artículo de Mirones Lozano, 2009.
} 
ñas del año 1280 se menciona a un judío de Corella al que llaman, "Ossaba” ('tío'), que ha de pagar una multa "porque crebanto paramento que avian feito los judios entre sí". 22

Por último, un testimonios acaso menos claro, pero no por ello inverosímil es el del apellido "Bete", relleno, con tendencia a engordar, quizás 'gordo' ${ }^{23}$ se menciona en 1381 a la mujer de "Salamon Bete" vendiendo una viña en la Barrena al racionero de la iglesia de Santa María de Tudela (Navarra Judaica, 3**, 753-10). Años más tarde, en 1407 aparece en el libro de cuentas del tesorero del rey un tal "Salamon Gordo" (Leroy y García Arenal, 1984: 236). No es un caso tan evidente de correspondencia entre el apellido en euskera y en romance como los mencionados anteriormente, puesto que "Bete" no es la palabra con la que normalmente se designa 'gordo' en euskera. Sin embargo, puesto que "Bete" no significa nada en hebreo ni en romance, ni se trata tampoco de un topónimo, se ha considerado oportuno mencionarlo como parte del repertorio de apellidos en lengua vasca que se recoge en este estudio.

\footnotetext{
${ }^{22}$ Navarra Judaica, 1, 123-19. Ejemplos de cristianos así apellidados: "Pero Osava” en 1247 y el correspondiente en romance: doña Franca del Tío (véase Michelena, 1969: 50). Se encuentran otros cristianos así apellidados en el Archivo Parroquial de San Cernín: "Johan Peritz de Ossaua", bureller mencionado en tres ocasiones con otros como testigo de un testamento en 1340 (García Larragueta, 1976).

${ }^{23}$ Michelena, 1987: s.v bete: 'llenar'; s.v. betea: (como participio en función de adjetivo) 'harto, saciado, relleno, gordo'.
} 


\section{Conclusiones}

El valor incalculable que pueden tener los nombres por sí mismos, lo que pueden sugerir y lo que pueden enseñar es indiscutible. Decía Albert Dauzat, con mucho acierto, que el valor mágico que se atribuía a las palabras en las sociedades antiguas era especialmente consistente en el caso de los nombres que designan a un individuo. Explicaba que, por ejemplo, los romanos estaban convencidos de que el nombre que se le pusiera a un niño ejercería sin duda una influencia sobre él, y por esta razón, los romanos cuando llamaban a un niño por ejemplo Fortis no lo hacían "parce que le nouveau né semblait courageux mais pour qu'il le devint” (1950: 7). También los judíos consideraron siempre la importancia de elegir un nombre apropiado en este sentido, pudiendo este determinar en parte el carácter del niño o su mismo destino (Talmud Babilonia, Ber. 7b). Por ejemplo los nombres dados en la Biblia revelan en la mayor parte de los casos un profundo significado ligado al momento del nacimiento del niño, la esperanza puesta por los padres en el recién nacido, o simplemente una expresión de gracias a Dios por su llegada al mundo de un nuevo ser, pero en ningún caso pondrán un nombre de forma arbitraria. ${ }^{24}$

\footnotetext{
${ }^{24}$ En su trabajo sobre antroponimia Benzion Kaganoff habla de una serie de decretos rabínicos emitidos con el fin de que se diera un nombre "purely Jewish" a todo niño nacido en el seno de una familia judía. Un nombre sagrado, en el momento de la circuncisión, y si acaso se utilizara otro no-judío con fines civiles. Esta medida se debía a que se había extendido enormemente el uso de nombres no-judíos desde el siglo xII. No era la primera vez que ocurría, y de hecho, este estatuto rabínico es válido hasta nuestros días. De algún modo muestra que nunca se olvida la importancia del nombre (1977: 49).
} 
Es bien conocido también que los árabes creen que el nombre que dan a sus hijos habrá de condicionar su forma de ser (Buendía, 2009). La lista de todas las civilizaciones en las que la antroponimia tienen un lugar de peso e importancia como para pensar que llevar un nombre es algo más que diferenciarse del vecino que se llama del mismo modo es muy larga.

En este estudio examinamos una época y una región donde quizás estos valores no tenían ya tanto peso. De cualquier modo, nombrar a alguien sigue siendo importante, quizás por razones distintas de las que venimos de mencionar. Cuando los judíos de los que hemos hablado se deciden por la forma eusquérica, no parece en ningún caso que estén heredando sin más un sobrenombre cuyo significado desconocen. Los sobrenombres se forman normalmente de forma espontánea y haciendo uso de la lengua de uso común. Su formación está ligada estrechamente a la lengua hablada.

Es cierto que puede ocurrir que una persona lleve un apellido cuyo significado desconoce; puede tratarse de un término en desuso, o corrompido; o bien un nombre heredado después de muchas generaciones y cuyo significado primero, si es que lo tuvo, se ha perdido hace tiempo. Lo mismo podría ocurrir con un apodo, también se puede heredar y perder en el camino su primer significado. Pero no es lo normal. Si el apodo que le asignan a uno tiene un significado concreto, si se trata de una palabra comprensible y en uso, es improbable que su portador no lo entienda a la perfección.

Si admitimos que los judíos referidos saben seguramente lo que sus sobrenombres vascos quieren decir, parece razonable afirmar que estaban en contacto estrecho con la 
lengua vasca, que les era familiar. Sin hacer afirmaciones irrefutables, es razonable pensar que si los judíos se comunicaban con sus vecinos cristianos en las lenguas que estos utilizaban ¿por qué no habrían de hablar vasco si encontraban que era necesario?

De momento es una pregunta sin respuesta. Sin embargo, el hecho documental es importante. La falta obvia de documentos suficientes que constaten de forma certera cualquier respuesta convincente no resta calidad a la reflexión. Sirva esta al menos para ampliar la perspectiva desde la que nos acercamos a esta minoría. Cualquier estudio de este tipo realizado en arreglo a una documentación determinada será siempre una muestra de la realidad muy fragmentaria, no siempre susceptible de ser completada. Si bien siempre se puede esperar que futuras investigaciones aclaren en parte lo que aquí tan solo se apunta.

Así pues, pese a las limitaciones inherentes a un objeto de estudio circunscrito a unas coordenadas espacio-temporales limitadas, este estudio ha puesto de manifiesto las confluencias, interferencias y tensiones lingüísticas de la Navarra judía bajo medieval. De este modo, se contribuye significativamente a dibujar un mapa algo más matizado y preciso de la realidad judía de la época haciendo uso de un elemento raramente considerado en este tipo de estudios: las concomitancias onomásticas. 


\section{Bibliografía}

Alegría, D. (2002), "Una anotación en euskera en las cuentas del reino de Navarra a finales del siglo xiII", Fontes Linguae Vasconum: Studia et Documenta, 89, pp. 139148.

Assis, Y. T. y J. R. Magdalena Nom de Deu y C. Lleal (1992), Judeolenguas marginales en Sefarad antes de 1492 (Aljamía romance en los documentos hebraiconavarros-siglo XIV), Barcelona, Universidad de Barcelona. BeCKer, L. (2009), "Names of Jews in Medieval Navarre (13th14th centuries)", en Names in Multi-Lingual, Multi-Cultural and Multi-Ethnic Contact: Proceedings of the 23rd International Congress of Onomastic Sciences, August 1722, 2008, York University, Toronto, pp. 140-156.

Buendia, P. (2009), "Del nombre árabe y sus misterios. Especulaciones y creencias sobre las propiedades augurales de la onomástica árabe", Estudios de Asia y África, XLIV (2), pp. 265-282.

Carrasco, J. (1993), Sinagoga y mercado. Estudios y textos sobre judíos del Reino de Navarra, Pamplona, Gobiernos de Navarra, Departamento de Cultura y Educación. Cierbide Martinena, R. (1996), “Onomástica de los judíos navarros (siglo xIv)", Nouvelle revue d'onomastique, 27-28, pp. 97-108.

Cierbide Martinena, R. et al. (2005), "La onomástica en Navarra y su relación con la de España”, en José Luis Ramírez Sádaba (coord.), Actas de las primeras Jornadas de Onomástica, (Pamplona, 2003), Navarra, Universidad Pública de Navarra. 
Dauzat, A. (1950), Les noms de personnes. Origine et évolution, París, Delagrave.

Gampel, B. R. (1989), The Last Jews on Iberian Soil: Navarrese Jewry 1479-1490, Berkeley, University of California Press.

García-Arenal, M. (1982), “Documentos árabes de Tudela y Tarazona", Al-Qantara, Revista de Estudios Árabes, 3, pp. 27-72.

García Larragueta, S. (1976), Archivo Parroquial de San Cernín de Pamplona. Colección diplomática hasta 1400, Pamplona, Diputación Foral de Navarra: Institución Príncipe de Viana.

Gold, D. S. (2003), "We do not know wether Jews in Sefarad 1 spoke or wrote Basque", Fontes Linguae Vasconum: Studia et Documenta, 94, pp. 537-540.

Gran enciclopedia de Navarra (1990), Pamplona, Caja de Ahorros de Navarra <http://www.enciclopedianavarra.com>

Haritschelhar, J. (ed.) (1983), Etre Basque, Toulouse, Privat.

Irigaray, A. (1970), "La carta eusquérica de 1415”, Fontes Linguae Vasconum: Studia et Documenta, 4, pp. 135-136.

_ (1960), "Onomástica medieval de Navarra”, Príncipe de Viana, XXI, 78-79, pp. 131-135.

IdoATE, F. (1969), "Una carta del siglo Xv en vascuence”, Fontes Linguae Vasconum: Studia et Documenta, 2, pp. 287-290. (1964), Catálogo documental de la ciudad de Corella, Pamplona, Aramburu.

Jimeno Jurío, J. M. (1997a), Navarra. Historia del Euskera, Tafalla, Txalaparta. 
Jimeno Jurío, J. M. (1997b), “Cirauqui (Navarra): pueblo monolingüe vasco", Fontes Linguae Vasconum: Studia et Documenta, 75, pp. 219-232.

Kaganoff, B. C. (1977), Dictionary of Jewish Names and Their History, Nueva York, Rowman \& Littlefield Publishers.

Lacarra, J. M. (1930), “Onomástica del siglo xiII", Revue Intenationel des Études Vasques, 21, pp. 247-254.

Lacave, J. L. (1998), Los judíos del reino de Navarra. Documentos hebreos 1297-1486, Pamplona, Institución Príncipe de Viana (Navarra Judaica, 7).

Lafon, R. (1973), La langue basque, Bayonne, Bulletin du Musée Basque.

Leroy, B. (2001), Les Menir, une famille sépharade à travers les siècles (xıIe-Xхе siècle), $2^{a}$ ed., Anglet, Atlantica (1 ${ }^{\text {a }}$ ed. París 1985, CNRS).

(1991), Los judíos de Navarra en la Baja Edad Media, Madrid, Dykinson.

_ (1978), "Le royaume de Navarre et les juifs aux xiv y XV siècles entre I'acueil et la tolerante», Sefarad, 38-2, pp. 263-292.

Leroy, B. y M. García Arenal (1984), Moros y judíos en la Baja Edad.Media, Madrid, Hiperion.

Magdalena Nom de Deu, R. (1988-1989), "Judeorromances 'marginales' de Sefarad”, Miscelánea de Estudios Árabes y Hebraicos (Sección de Hebreo), 33-33/2, pp. 41-53.

Michelena Elissalt, K. (1987-2005), Diccionario general vasco, Bilbao, Desclée de Brouwer/Mensajero.

__ (1977), La lengua vasca, Durango, Leopoldo Zugaza. 
Mirones Lozano, E. (1999), Los judíos del Reino de Navarra en la crisis del siglo XV (1425-1479), Pamplona, Gobierno de Navarra.

_ (2009), "Mehe: un apellido vasco escrito en aljamía hebraico-navarra", Fontes Linguae Vasconum: Studia et Documenta, 110, pp. 117-124.

Niremberg, D. (2001), Comunidades de violencia: persecución de minorías en la Edad Media, Barcelona, Península.

Orpustan, J. B. (2000), “Anthroponomastique médiévale en Pays basque: Prenoms et surnoms en Basse-Navarre et Soule au début du xive siècle (1305-1350)", Lapurdum [online], 5.

_ (1997), "Histoire et onomastique médiévales. L’enquête de 1249 sur la guerre de Thibaud I de Navarre en Labourd", Lapurdum [online], 2.

Riera I Sans J. (2008), "Cent trenta-nou volums de llibres d'un jueu mercader i talmudista: Mossé Almaterí (1362)", Sefarad, 6, pp. 15-35.

(2009), “Jaime I y los judíos de Cataluña”, en Esteban Sarasa Sánchez (coord.), La sociedad en Aragón y Cataluña en el reinado de Jaime I, 1213-1276, Madrid, Institución Fernando el Católico.

Romano, D. (1980), "Los Surí, judíos de Huesca y perleros de la Reina de Aragón (1350-¿1372?)", Sefarad, 2, pp. 255-282.

Trask, R. L. (1997), The History of Basque, Londres, Routledge.

VAldeon, J. (2006), Cristianos, judíos y musulmanes, Barcelona, Crítica. 